\title{
Desempenho em uma tarefa de timing coincidente e velocidade do estímulo: o uso de índices de acertos
}

\author{
Performance in a coincidence timing task and stimulus velocity: \\ use of hit rates
}

\author{
Maria Teresa Cattuzzo \\ Luciano Basso \\ Rafael dos Santos Henrique \\ Jorge Sabino Pereira de Oliveira ${ }^{1}$
}

1 Universidade de Pernambuco. Escola Superior de Educação Física. Recife, PE. Brasil.

2 Universidade de São Paulo. Laboratório de Comportamento. São Paulo, SP. Brasil.

Recebido em 01/03/09 Revisado em 11/04/09 Aprovado em 22/09/09
Resumo - O objetivo deste estudo foi investigar o número de acertos consecutivos em uma tarefa complexa de timing coincidente, que foram utilizados como índices de desempenho, e o efeito da velocidade do estímulo luminoso no alcance desses índices. Trinta adultos de ambos os sexos foram testados no equipamento de Timing Coincidente para Tarefas Complexas em três velocidades do estímulo luminoso: alta, moderada e baixa. As análises mostraram que dois índices de desempenho podem ser usados como critério da estabilização do desempenho: três e quatro acertos consecutivos na meta de coincidência. A análise descritiva parece sugerir que na condição de alta velocidade o índice de acertos consecutivos talvez tivesse que ser mais rigoroso.

Palavras-chave: Aprendizagem; Prática; Desempenho Psicomotor.

Abstract - The aim of this study was to investigate the number of consecutive hits in a complex coincidence timing task, which were used as performance indices, and the effect of light stimulus velocity on reaching these indices. Thirty adults of both genders were tested using an apparatus of coincidence timing for complex tasks and three light stimulus velocities (high, moderate and low). Analysis showed that two performance indices can be used as criteria of performance stabilization: three and four consecutive hits in the coincidence goal. Descriptive analysis suggests that the index of consecutive hits should be more rigorous at high light stimulus velocity.

Key words: Learning; Practice; Psychomotor Performance. 


\section{INTRODUÇÃO}

Timing antecipatório, também denominado antecipação de coincidência ou timing coincidente é a capacidade perceptual-motora para executar uma resposta motora em sincronia com a chegada de um objeto externo, em um determinado ponto'; ele depende de processos de seleção, planejamento e execução para antecipar tanto a chegada do estímulo quanto a resposta efetora, de modo que ocorram simultaneamente ${ }^{2}$. Tradicionalmente, as medidas de desempenho usadas em estudos com tarefas de timing antecipatório são: o erro absoluto, que expressa a magnitude do erro; o erro constante, que expressa a direção do erro (respostas atrasadas ou adiantadas) e o erro variável, que expressa a consistência do desempenho ${ }^{3}$. Durante a prática, quando os erros absoluto, constante e variável diminuem é um indício que o sistema percepto-motor encontrou uma solução apropriada para resolver o seu problema de organização temporal, e tende a repetir essa solução.

Do ponto de vista do pesquisador, a melhora do desempenho tem um importante significado teórico, pois a diminuição do erro e da sua variabilidade indicam uma mudança funcional no desempenho. Do ponto de vista do sujeito, vale questionar se esse dado traz significado, pois Schmidt e Lee ${ }^{3}$ afirmam que tanto em termos filosóficos quanto educacionais, na maioria dos aspectos da vida, julgamo-nos não com base na melhora do nosso desempenho, mas com base sobre "o quê" efetivamente conseguimos fazer.

Também, nos métodos tradicionais utilizados para investigar a aprendizagem, é comum estabelecer certa quantidade de tentativas que deve ser praticada para possibilitar a estabilização do desempenho. Mas um problema inevitável decorre desse procedimento: a negligência às diferenças individuais com relação à quantidade de prática necessária para atingir essa estabilização. Drowatzky já afirmava que as curvas individuais mostram amplas variações entre sujeitos, sendo que, em algumas, nota-se aumentos graduais e/ou estirões súbitos e, em outras, aceleração negativa. Ele concluiu que o progresso no comportamento parece ser de natureza mais errática do que regular, e as curvas médias escondem essas diferenças individuais.

Portanto, para todo indivíduo engajado em aprender uma tarefa, a prática é fundamental, mas isso não significa que todos necessitem da mesma quantidade, por exemplo, um estudo com uma tarefa de timing coincidente evidenciou que crianças (10 a 12 anos) necessitaram de 3 até 136 tentativas para atingirem a meta de coincidência.
No modelo teórico do Processo Adaptativo ${ }^{6-10}$, a aprendizagem motora é um processo contínuo e cíclico que leva o sistema ao aumento de complexidade: $\mathrm{o}$ sistema se torna funcionalmente apto a responder a demandas futuras; esse processo é composto de, pelo menos, duas fases: a de formação da estrutura mental e a de adaptação dessa estrutura; a passagem de uma fase para outra é feita mediante a resposta à perturbação inserida no sistema. Assim, aprendizagem é uma mudança funcional, inferida pelo desempenho, que capacita o sujeito a alcançar a meta da tarefa, mesmo sob novas demandas ambientais/informacionais. Nessa abordagem, a formação da estrutura mental é o primeiro passo do processo e obter indicativos objetivos para a inferência dessa formação é, sem dúvida, uma tarefa que exige atenção metodológica.

O uso de critérios de desempenho para inferir a aprendizagem é um recurso metodológico usado desde os primeiros estudos da área de aprendizagem motora. De acordo com Adams ${ }^{11}$, McGeogh e Melton, em 1929, já era usado o critério de executar uma tentativa sem erro em tarefas verbais e motoras, para inferir a aprendizagem.

Muitos critérios de desempenho podem ser estabelecidos, mas é essencial que eles atendam à teoria de base do estudo em questão. Um critério de desempenho que pode ser bem aproveitado, quando se investiga aprendizagem motora como um processo adaptativo, são os índices de acertos contínuos: ao realizar um dado número de sucessos consecutivos, no alcance da meta da tarefa, infere-se que o sistema está num nível funcionalmente diferente, sendo capaz de responder com um padrão espaço-temporal consistente e após isso, o sistema poderia ser submetido às variações ambientais/informacionais.

O índice de acertos vem sendo usado como critério de estabilização do desempenho em tarefas de timing coincidente na abordagem do Processo Adaptativo. No estudo de Corrêa ${ }^{12}$, o índice foi a realização de três execuções consecutivas com erro inferior a $30 \mathrm{~ms}$. Posteriormente, outros estudos também adotaram o mesmo índice ${ }^{13-15}$.

Antes de estabelecer um índice de acertos numa tarefa de timing coincidente, poder-se-ia questionar "quantos" acertos consecutivos ocorrem ao longo das tentativas de prática. Também seria importante testar se esses índices de acertos consecutivos sofrem efeito da velocidade do estímulo visual, uma vez que os estudos sobre timing coincidente têm mostrado resultados contraditórios: alguns evidenciaram que os melhores desempenhos em tarefas de timing coincidente ocorriam em velocidades consideradas altas ${ }^{16-19}$, mas em outros, ocorriam em velocidades intermediária ${ }^{20} \mathrm{Ou}$ 
baixas $^{21}$, e alguns estudos indicaram desempenhos similares em diferentes velocidades ${ }^{22-24}$.

Assim, o presente trabalho se propos a: (a) quantificar os acertos consecutivos em uma tarefa complexa de timing coincidente em função da quantidade de prática; (b) discutir quantos acertos consecutivos poderiam ser usados como índices de desempenho e (c) testar o efeito da velocidade do estímulo visual na obtenção desse(s) índice(s).

\section{PROCEDIMENTOS METODOLÓGICOS}

\section{Amostra}

Nessa pesquisa quase-experimental, trinta adultos foram recrutados durante um curso de formação continuada em Educação Física, na Universidade de Pernambuco (Recife/PE) e distribuídos em grupos de dez participantes cada, que se distinguiam pela velocidade do estímulo visual: G1 - velocidade rápida $(1,7 \mathrm{~m} / \mathrm{s})(3$ mulheres e 7 homens; $29,9 \pm 4,93$ anos); G2 - velocidade intermediária $(1,5 \mathrm{~m} / \mathrm{s})(3$ mulheres e 7 homens; $28,7 \pm 5,07$ anos) e G3 - velocidade lenta $(1,3 \mathrm{~m} / \mathrm{s})$ (4 mulheres e 6 homens; 29,9 anos $\pm 6,5$ anos). As velocidades foram adaptadas de estudos com esse tipo de tarefa ${ }^{23,24}$. Os participantes foram devidamente informados e assinaram o termo de participação voluntária em pesquisa.

\section{Equipamento e tarefa}

$\mathrm{O}$ equipamento utilizado foi o de Timing Coincidente para Tarefas Complexas (P.I. 0.403.433-4, de 03/08/2004). Detalhes do equipamento podem ser visto em Corrêa et $\mathrm{a}^{23}$. A tarefa consistiu em tocar sensores em uma ordem, sendo que o toque no último sensor deveria coincidir com o acendimento do último de uma série de diodos dispostos em frente ao sujeito. Um dispositivo com cinco diodos nas cores vermelha, amarela e verde que qualificavam o desempenho, foi usado para o Conhecimento de Resultados (CR). O acendimento do diodo vermelho, à direita, significava desempenho muito adiantado e à esquerda, muito atrasado. O diodo amarelo, à direita, significava desempenho um pouco adiantado e à esquerda, um pouco atrasado. $\mathrm{O}$ acendimento do diodo verde indicava o alcance da meta de coincidência que tinha uma margem de tolerância de \pm 30 ms.

\section{Procedimentos}

A tarefa e o dispositivo de CR eram explicados aos sujeitos, enfatizando que deveriam obedecer a ordem de toques e coincidirem o toque no último sensor com o acendimento do último diodo. Após duas/três tentativas para ajustarem-se ao equipa- mento, a coleta era iniciada.

$\mathrm{O}$ experimento constou das fases de aquisição e adaptação. $\mathrm{Na}$ fase de aquisição os sujeitos realizaram 150 tentativas, recebendo CR em cada uma delas; a sequência utilizada (1-2-4-3-5) foi semelhante aos trabalhos com essa mesma tarefa e equipamento ${ }^{23,24}$. $\mathrm{O}$ número de tentativas foi definido em estudos pilotos, nos quais foi possível observar a melhora do desempenho, sem fadiga ou perda de motivação. Para a fase seguinte - adaptação -, houve a solicitação de mais toques nos dois primeiros sensores (1-2-1-2-4-3-5); nessa fase foram executadas 30 tentativas sem CR. O intervalo entre as fases foi o tempo suficiente para a explicação sobre a modificação da tarefa.

\section{Medidas}

O desempenho foi avaliado pelo erro absoluto (diferença entre o tempo para acendimento de todos os diodos e o tempo para realizar todos os toques) e erro de execução (não tocar um sensor da sequência e/ou realizar a sequência incorreta). $O$ índice de acertos foi o número de vezes consecutivas que o sujeito atingia a meta. As medidas de desempenho foram computadas em blocos de 10 tentativas.

\section{Tratamento estatístico}

A análise exploratória constou da observação de outliers mediante a inspeção do diagrama de extremos e quartis. A análise da normalidade das distribuições foi realizada mediante a utilização do teste de Shapiro-Wilk. A falta de normalidade na maior parte dos blocos conduziu ao uso de testes não paramétricos e como medida de tendência central, a mediana. Para a análise entre os blocos, foi utilizado o teste de Friedman e para a análise intergrupos o teste de Kruskal-Wallis; para as multicomparações entre grupos e intrablocos, foram utilizados os testes sugeridos por Siegel e Castellan $\mathrm{Jr}^{25}$. O pacote estatístico utilizado foi o SPSS 13.0. Para a análise de proporções entre os grupos, foi utilizado o módulo Trend do software Pepi 4.0.

\section{RESULTADOS}

\section{O efeito da prática}

Na Figura 1, nota-se uma tendência de queda no erro de execução nos três grupos, mas, no último bloco da aquisição, ocorreu aumento para G2 e G3. Na adaptação, G1 e G3 mostraram aumento, enquanto que o G2 mostrou queda; no final da adaptação todos os grupos diminuem esse erro e situam-se no mesmo nível. 


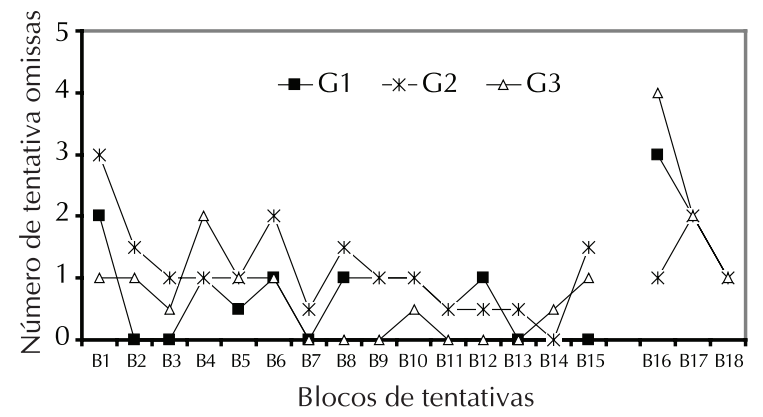

Figura 1. Valores da mediana do erro de execução nas fases de aquisição (do B1 até B15) e adaptação (do B16 a B18) para os três grupos.

A série de Friedman para a fase de estabilização não indicou diferença significativa entre os blocos em qualquer um dos grupos. No entanto, na adaptação houve diferenças significativas no G1 $\left[\chi^{2}(\mathrm{n}=5, \mathrm{gl}=3)=10,21 ; \mathrm{p}=0,017\right]$, entre o último bloco da estabilização e o último bloco da adaptação. Também houve diferença no G3 $\left[\chi^{2}(n=9\right.$, $\mathrm{gl}=3)=11,85 ; \mathrm{p}=0,008$, entre o último bloco da aquisição e os dois últimos da adaptação (do B15 para B17 e B18), e entre o primeiro e o último da adaptação (do B16 para o B18).

Os testes de Kruskal-Wallis não apontaram diferenças significativas tanto na estabilização quanto adaptação entre os grupos.

A Figura 2 mostra que os grupos diminuíram o erro absoluto na fase de aquisição e na adaptação, ocorreu aumento para os três grupos no B16, sendo que nos próximos blocos dessa fase, G2 e G3 diminuíram, e o G1 apresentou aumento no último bloco.

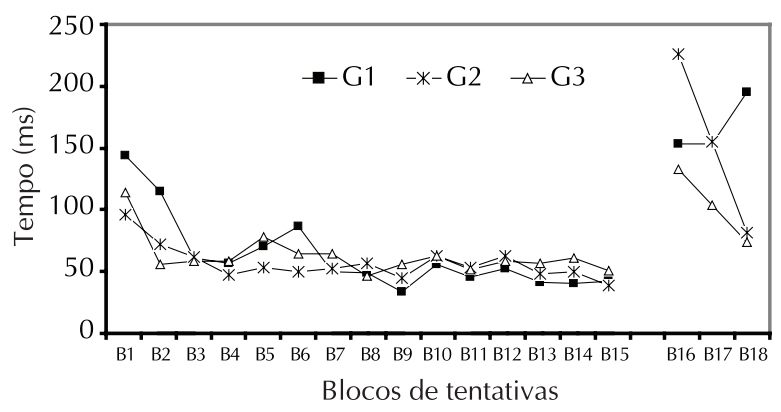

Figura 2. Valores da mediana do erro absoluto nas fases de aquisição (do B1 até B15) e adaptação (do B16 até B18) para os 3 grupos.

A série de testes de Friedman indicou diferenças significativas entre os blocos para o G1 $\left[\chi^{2}(\mathrm{n}=8, \mathrm{gl}=14)=28,41 ; \mathrm{p}=0,012\right], \mathrm{G} 2$ $\left[\chi^{2}(n=10, g l=14)=28,36 ; p=0,012\right]$ e $\mathrm{G} 3\left[\chi^{2}(n=9\right.$, $\mathrm{gl}=14)=33,57 ; \mathrm{p}=0,002]$. Os testes de múltiplas comparações indicaram que para o G1 as diferenças ocorreram entre o primeiro bloco e os demais; para o G2 ocorreram entre o B1 e B4 a B10, e para o G3 ocorreram entre o B1 e B8 a B10 e entre o B5 e B6 a B10. Já na adaptação somente houve diferenças significativas para o $\mathrm{G} 2\left[\chi^{2}(\mathrm{n}=10, \mathrm{gl}=3)=23,40 ; \mathrm{p}=0,003\right]$ e para o $\mathrm{G} 3\left[\chi^{2}(\mathrm{n}=9, \mathrm{gl}=3)=11,40 ; \mathrm{p}=0,009\right]$. Os testes de múltiplas comparações indicaram diferenças significativas para o G2 entre o B15 e B16, B16 e B18; e para o G3 ocorreram entre o B15 e B16. A série de análises intergrupos não identificou diferenças na aquisição nem na adaptação. Com isso, entende-se que os grupos não apresentaram comportamento diferente entre eles em nenhuma das fases.

\section{O índice de acertos consecutivos e a quantidade de prática.}

De modo geral, com a continuidade da prática, houve aumentos gradativos dos acertos consecutivos, mas os participantes apresentaram uma grande heterogeneidade, desde aqueles que atingiram a meta somente duas vezes até um que conseguiu atingir a meta 11 vezes consecutivas (Figura 3)

Nas 30 primeiras tentativas (20\% da aquisição), atingir a meta duas vezes consecutivas ocorreu para $70 \%$ dos sujeitos do G1, 100\% do G2 e 80\% do G3; $40 \%$ dos sujeitos do G1, e 50\% dos sujeitos do G2 e do G3 atingiram a meta três vezes consecutivas; somente 20\% dos sujeitos do G1 e G2 e 40\% dos sujeitos do G3 atingiram a meta quatro vezes consecutivas; apenas um sujeito do G3 atingiu a meta 11 vezes consecutivas.

Com 60 tentativas (40\% da aquisição), a proporção de sujeitos que atingiu a meta duas, três e quatro vezes consecutivas aumentou, o que ficou mais evidente no G3; no G1, 20\% dos sujeitos atingiram a meta cinco vezes consecutivas e no G2 um sujeito atingiu a meta sete vezes consecutivas.

Com 90 tentativas (60\% da aquisição), no mínimo, $70 \%$ dos sujeitos dos três grupos atingiram a meta três vezes consecutivas; $40 \%$ ou mais dos sujeitos dos três grupos atingiram a meta quatro vezes consecutivas e pelo menos um sujeito de cada grupo atingiu a meta seis vezes consecutivas.

Com 120 tentativas (80\% da aquisição), foi mantida a proporção de sujeitos que atingiu a meta três vezes consecutivas; além disso, pelo menos um sujeito de cada grupo atingiu a meta sete vezes consecutivas.

Com 150 tentativas, o G2 aumentou a proporção de sujeitos que atingiram a meta três, quatro, cinco e seis vezes consecutivas; houve aumento na proporção de sujeitos que atingiram a meta sete vezes consecutivas no $\mathrm{G} 2$ e no G3; somente um sujeito do G2 atingiu a meta 10 vezes. 

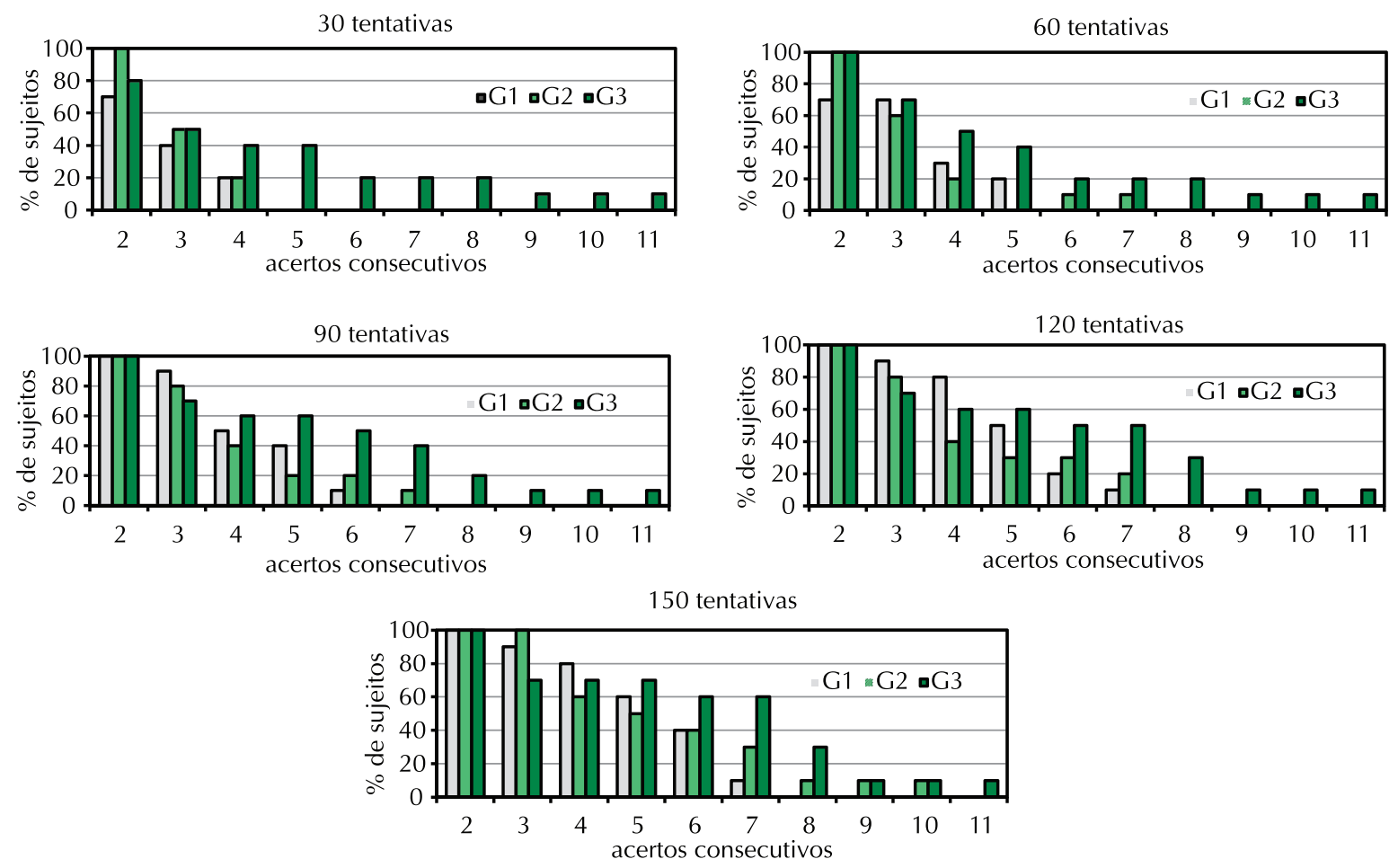

Figura 3. Percentual acumulativo de sujeitos em função do número de acertos consecutivos em 30, 60, 90, 120 e 150 tentativas de prática.

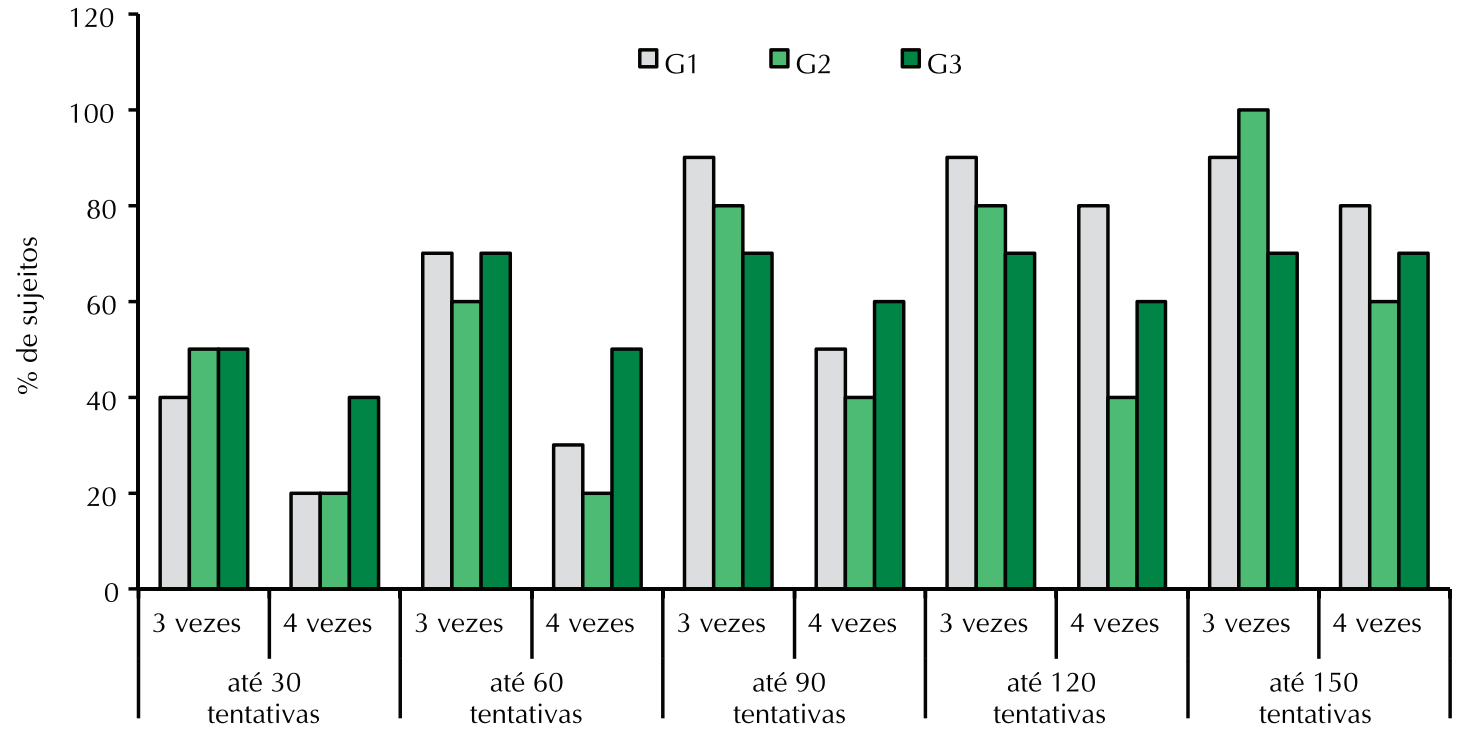

Figura 4. Porcentagem acumulada de sujeitos que acertam três e quatro vezes consecutivas ao longo das tentativas da aquisição.

O efeito da velocidade em função da obtenção dos índices de acertos consecutivos da meta ao longo das tentativas

Na observação da Figura 4, pareceu haver diferenças na obtenção de três e quatro acertos consecutivos em função da velocidade, principalmente, no início da prática.

Foi testado o efeito da velocidade do estímulo visual no momento da primeira obtenção de três e quatro acertos consecutivos. $\mathrm{O}$ teste de Kruskal-
Wallis não identificou diferenças significativas tanto para 3 acertos $\left[\chi^{2}(n=26, g l=2)=1,48 ; p=0,47\right]$ quanto para 4 acertos consecutivos $\left[\chi^{2}(n=22\right.$, $\mathrm{gl}=2)=0,38 ; \mathrm{p}=0,82]$.

A inspeção visual da Figura 4 também evidenciou um aumento brusco de quatro acertos consecutivos no G1, entre 90 e 120 tentativas e, então, foi testado se ocorria diferença significativa entre os grupos, mas isso não foi confirmado estatisticamente pelo teste de múltiplas comparações do módulo Trend. 


\section{DISCUSSÃO}

De modo complementar, a análise do efeito da prática e a análise dos acertos consecutivos evidenciaram as mudanças funcionais no desempenho. Obter sucesso duas vezes consecutivamente foi considerado um requerimento de baixa magnitude e, consequentemente, parece ser um índice pouco rigoroso para ser usado como critério de estabilização do desempenho. Para atingir três acertos consecutivos, 70\% ou mais dos participantes, em todos os grupos, necessitaram de até 90 tentativas. Esse parece ser um índice de acertos mais aceitável, pois foi desafiante (não se alcança rapidamente), mas realizável pela maioria dos sujeitos. Atingir a meta quatro vezes consecutivas pode ser considerado um índice mais rigoroso, pois exigiu um maior número de tentativas (150) para que 60 a $80 \%$ de sujeitos pudessem alcançá-lo. $O$ índice de atingir a meta cinco vezes parece ser muito rigoroso, pois necessitou das 150 tentativas para que, pelo menos, $50 \%$ dos sujeitos de todos os grupos o alcançassem.

Até 60 tentativas, não houve efeito do aumento da velocidade do estímulo visual na obtenção de três e quatro acertos consecutivos, mas a partir daí a superioridade do G1 em realizar acertos consecutivos levou ao questionamento se a velocidade rápida teria efeito sobre esse critério de desempenho a partir dessa quantidade de prática. Essa diferença não se confirmou estatisticamente. Poder-se-ia especular que: a) as velocidades utilizadas no presente estudo não foram suficientemente altas ou baixas para serem discriminadas como tal, e o aumento de 30\% na magnitude da velocidade não provocou mudanças significativas no desempenho ou b) que o critério de três acertos consecutivos não seria suficiente para atestar o novo estado do sistema

Passou-se a questionar o efeito do aumento da velocidade para o critério de quatro vezes consecutivas, evento visualmente bem evidenciado na Figura 4, a partir da $90^{a}$ tentativa, e isso poderia sugerir que o sistema se encontrava num outro estado, demarcado por um critério mais rigoroso. No estudo de Engstrom et al. ${ }^{26}$, o desempenho de timing coincidente foi melhor para velocidades mais altas do que para mais baixas e eles sugeriram que estímulos mais rápidos levam o sistema a mudar para um modo antecipatório, evitando atrasos desnecessários. No entanto, no presente estudo, o resultado descritivo não foi confirmado inferencialmente.

Com relação à quantidade de prática, num estudo com uma tarefa simples de timing coincidente, Freudenheim ${ }^{27}$ afirmou ser indiferente para adultos praticar o número de tentativas necessário à esta- bilização ou mais, e questionou se a quantidade de prática seria diretamente determinada pelo número de tentativas. Assim, poder-se-ia especular se o desempenho é proporcional ao aumento de tentativas. Fundamentado nos resultados do presente estudo, pode-se dizer que houve uma proporcionalidade entre o aumento da quantidade de prática e o número de acertos consecutivos, mas isso não significa uma proporcionalidade na mudança do estado do sistema.

Embora tenham sido verificadas diferentes quantidades de acertos consecutivos ao longo das tentativas, os resultados mostraram que, considerando as três velocidades, 90 tentativas possibilitaram alcançar um nível "ótimo" de desempenho. Esses resultados e interpretação dão suporte ao estudo de Corrêa ${ }^{5}$ que, utilizando essa mesma tarefa de timing coincidente em crianças (10 a 12 anos), com velocidade de $142,2 \mathrm{~cm} / \mathrm{s}$, mostrou que aquelas que alcançaram o critério de três acertos consecutivos numa faixa de erro de $50 \mathrm{~ms}$, entre 71 e 80 tentativas e acima de 91 tentativas, eram as que tinham o comportamento mais consistente.

\section{CONCLUSÃO}

Uma vez que a natureza do processo de aprendizagem não está totalmente estabelecida, nem foram ainda estudados todos os fatores cruciais que interferem na aprendizagem, seria importante estabelecer critérios que evidenciassem a mudança funcional do desempenho e que atendessem a especificidade de cada indivíduo.

Essa investigação sobre índice de desempenho na aprendizagem de uma tarefa de timing em adultos permitiu observar que três e quatro acertos consecutivos podem ser usados como índices que, por sua vez, permitem inferir que o sistema atingiu um determinado estado de competência; no entanto, em termos operacionais, nos experimentos de aprendizagem é importante definir quanta prática é necessária para atingir tais índices. Nesse caso, sugere-se que para essa amostra e nessa tarefa complexa de timing coincidente, 90 tentativas foram consideradas uma quantidade adequada.

Ainda que não tenha existido confirmação estatística da diferença entre os grupos, os resultados descritivos permitem que se deixe em aberto a questão de que, em velocidades mais altas, o critério de acertos talvez devesse ser mais rigoroso.

\section{REFERÊNCIAS BIBLIOGRÁFICAS}

1. Beslile JL. Accuracy, reliability and refractoriness in a coincidence-anticipation task. Res Q Exerc Sport 1963;34:271-281. 
2. Tresilian JR. Perceptual and cognitive process in time-to-contact estimation. Percept Psychophys 1995; 57(2):231-245.

3. Schmidt RA, Lee TD. Motor control and learning: a behavioral emphasis. $3^{a}$ ed. Champaign: Human Kinetics, 1999.

4. Drowatzky JN. Motor learning. Principles and practices. $2^{\mathrm{a}}$ ed. Minneapolis, Minnesota: Burgess, Pub Co, 1981.

5. Corrêa UC. Timing coincidente e diferenças individuais: efeitos da quantidade de prática no desempenho de crianças. In: Barreiros J, Cordovil R, Carvalheiro $\mathrm{S}$, organizadores. Desenvolvimento motor da criança. Lisboa: FHM Edições; 2007. p.115-124.

6. Tani G. Hierarchical organization of an action program and the development of skilled actions. Unpublished Technical Report. Sheffield: Department of Psychology of University of Sheffield, 1995.

7. Tani G. Processo adaptativo na aprendizagem de uma habilidade motora. [Tese de Doutorado em Educação]. Faculdade de Educação da Universidade de Hiroshima; 1982 (em japonês).

8. Tani G. Variabilidade de resposta e processo adaptativo em aprendizagem motora. [Tese de Livre Docência]. Escola de Educação Física e Esporte da Universidade de São Paulo, 1989.

9. Tani G. Processo adaptativo: uma concepção de aprendizagem motora além da estabilização. In: Tani G. editor. Comportamento motor: Aprendizagem e desenvolvimento. Rio de Janeiro: Guanabara Koogan, 2005.

10. Tani G, Bastos FC, Castro IJ, Jesus JF, Sacay RC, Passos SCE. Variabilidade de resposta e processo adaptativo em aprendizagem motora. Rev paul Educ Fís 1992;6(1):16-25.

11. Adams JA. Historical review and appraisal of research on the learning, retention, and transfer of human motor-skills. Psychol Bull 1987;101(1) 41-74.

12. Corrêa UC. Estrutura de prática e processo adaptativo na aquisição de habilidades motoras. [Tese de Doutorado]. Escola de Educação Física e Esporte da Universidade de São Paulo,2001.

13. Ugrinowitsch H. Efeito do nível de estabilização do desempenho e do tipo de perturbação no processo adaptativo em aprendizagem motora. [Tese de Doutorado]. Escola de Educação Física e Esporte da Universidade de São Paulo, 2003.

14. Paroli R. Efeito da estrutura de prática na aquisição de uma habilidade motora. [Dissertação de Mestrado]. Escola de Educação Física e Esporte da Universidade de São Paulo, 2004.

15. Corrêa UC, Gonçalves LA, Barros JAC, Massigli M. Prática constante-aleatória e aprendizagem motora: efeitos da quantidade de prática constante e da manipulação de exigências motoras da tarefa. Brazilian J Mot Behav 2006;1(1):41-52.
16. Wrisberg CA, Hardy CJ, Beitel PA. Stimulus velocity distance as determiners of movement velocity and coincident timing accuracy. Hum Factors 1982;24(2):599-608.

17. Meeuwsen HJ, Goode SL, Goggin NL. Effects of aging on coincidence anticipation timing in females. J Aging Phys Act 1997;5(4):285-297.

18. Durham PJ, Reeve J. Sex, eye preference and speed of stimulus effect on anticipation of coincidence. Percept Mot Skills 1990;71(3):1172-1176.

19. Williams LRT. Coincidence timing of a soccer pass: effects of stimulus velocity and movement distance. Percept Mot Skills 2000;91(1):39-52.

20. Teixeira LA, Santos VA, Andreysuk R. Tarefas que envolvem timing antecipatório: seriam as velocidades mais baixas as mais fáceis para sincronizar? Rev paul Educ Fís 1992;6(2):21-28.

21. Hart MA. Transfer of participation in fast - pitch softball to the performance of a coincidence antitcipation task. J Hum Mov Stud 2004;46(5):359-371.

22. Harrold D, Kozar B. Velocity, occlusion and sex of subjects in coincidence of anticipation. Percept Mot Skills 2002;94(3):914-920.

23. Corrêa, UC, Oliveira, P H, Oliveira, J.A, Freudenheim, AM, Paroli, R, Ugrinowitsch, et al. Timing coincidente em tarefas complexas: estudo exploratório do desempenho de adultos de diferentes idades em diferentes velocidades de estímulo visual. Rev bras Educ Fís Esp 2005;19(4):307-315.

24. Freudenheim, AM, Oliveira, JA, Corrêa, UC, Oliveira, PH, Dantas, LEPBT, Silva, J.A, et al. Efeito da velocidade do estímulo no desempenho de uma tarefa de timing coincidente em crianças e adolescentes Rev Port Cien Desp 2005; 5(2):160-166.

25. Siegel S, Castellan Jr NJ. Estatística não-paramétrica para ciências do comportamento. $2^{a}$ edição. São Paulo: Bookman, 2006.

26. Engstrôm DA, Kelso JAS, Holroyd T. Reactionanticipation transitions in human perception-action patterns. Hum Mov Sci 1996;15(6):809-832.

27. Freudenheim AM. Um teste à formação de esquema: efeito da variabilidade e da quantidade de prática na produção de movimentos novos em adultos. Rev paul Educ Fís 1994;8(1):3-16.
Endereço para correspondência
Maria Teresa Cattuzzo
Rua Bispo Coutinho 821 - Carmo.
CEP: 53120-130 - Olinda - PE
E-mail: mtcattuzzo@hotmail.com 